\title{
A class of implicit symmetric symplectic and exponentially fitted Runge-Kutta-Nyström methods for solving oscillatory problems
}

\author{
Huai Yuan Zhai ${ }^{1}$, Wen Juan Zhai ${ }^{2}$ and Bing Zhen Chen ${ }^{3^{*}}$ [D
}

\section{"Correspondence:}

chenbingzhen6026@163.com

${ }^{3}$ School of Science, Beijing Jiaotong

University, Beijing, China

Full list of author information is

available at the end of the article

\section{Springer}

\begin{abstract}
The construction of implicit Runge-Kutta-Nyström (RKN) method is considered in this paper. Based on the symmetric, symplectic, and exponentially fitted conditions, a class of implicit RKN integrators is obtained. The new integrators called ISSEFMRKN integrate exactly differential systems whose solutions are linear combinations of functions from the set $\{\exp (\lambda t), \exp (-\lambda t), \lambda \in \mathbb{C}\}$. In addition, their final stages also preserve the quadratic invariants $\{\exp (2 \lambda t), \exp (-2 \lambda t)\}$. Especially, we derived two methods: ISSEFMRKNs 102 and ISSEFMRKNs204 which are of order 2 and 4 , respectively. And the method ISSEFMRKNs204 has variable nodes. The derived method ISSEFMRKNs204 reduces to the classical RKN method (Qin and Zhu in Comput. Math. Appl. 22(9):85-95, 1991) as $\lambda h \rightarrow 0$. The numerical results show that our methods possess the efficiency and competence compared with some implicit RKN methods in the literature. Especially, ISSEFMRKNs204 improves the accuracy compared with unmodified method ISSEFRKNs204 proposed in (Zhai and Chen in Numer. Algebra Control Optim. 9(1):71-84, 2019).
\end{abstract}

MSC: 65L05; 65L06; 65M20; 65N40; 49M15

Keywords: Implicit; Symmetric; Symplectic; Exponentially fitted; Large step

\section{Introduction}

In this paper we focus on the initial value problems (IVP) related to systems of secondorder ODEs of the form

$$
y^{\prime \prime}=f(t, y), \quad y\left(t_{0}\right)=y_{0}, \quad y^{\prime}\left(t_{0}\right)=y_{0}^{\prime}, \quad t \in\left[0, t_{\text {end }}\right],
$$

whose solutions exhibit an oscillatory character. In applied sciences, such as molecular dynamics, orbital mechanics, and electronics, many problems of this type arise. For these problems, high accuracy of integration is often the first requirement. There are many researchers focusing on this problems. Until now there have been broadly two categories of approaches to numerical integration of IVP (1): indirect and direct. On the one hand, if we introduce a new variable $u$ to represent the first derivative $y^{\prime}$, then IVP (1) comes into

(c) The Author(s) 2018. This article is distributed under the terms of the Creative Commons Attribution 4.0 International License (http://creativecommons.org/licenses/by/4.0/), which permits unrestricted use, distribution, and reproduction in any medium, provided you give appropriate credit to the original author(s) and the source, provide a link to the Creative Commons license, and indicate if changes were made. 
a partitioned system of first-order equations

$$
y^{\prime}=u, \quad u^{\prime}=f(t, y), \quad y\left(t_{0}\right)=y_{0}, \quad u\left(t_{0}\right)=y_{0}^{\prime} .
$$

Thus it can be solved by the general purpose Runge-Kutta (RK) methods or partitioned Runge-Kutta (PRK) methods (see Refs. [4, 5, 21, 22, 28, 29]). One disadvantage of this procedure is that we need to solve a problem with more variables than the original problem. Therefore, in 1925 Nyström designed a method called Runge-Kutta-Nyström (RKN) method to deal with the second-order problem (1) directly. Since then, plenty of studies have been dedicated to the design of RKN methods. What followed are some variations of the standard RKN methods, such as $[6,9,16,23,24,27,30,31]$ and so on.

As is known, the symplectic Runge-Kutta-Nyström methods share the pronounced property of being zero-dissipative, which is an important requirement for solving oscillatory problem. Simos and Vigo-Aguiar [26] then considered symplectic methods of RKN type which are adapted to certain types of oscillators. The term symplecticity essentially means area preserving in a phase space. Approximate solutions generated by symplectic methods are conservative even at finite resolution, in contrast with numerical methods that generate approximate solutions that are conservative only in the limit as the time step size approaches zero. Symplectic methods have been applied to many problems such as pendulum, Morse oscillator, harmonic oscillator, Lennard-Jones oscillator, Kepler's orbit problem, and so on. In addition, it is pointed out in Chap. V and Chap. XI of [10] that symmetric methods show a better long time behavior than non-symmetric ones when applied to reversible differential systems, as it is the case for conservative mechanical systems. So, some symmetric and symplectic RKN methods have been proposed such as [20].

However, they do not consider exponential fitting conditions. Exponentially fitted methods share very good behaviors when the solution of the problem can be expressed as linear combinations of functions from $\{\exp (\lambda t), \exp (-\lambda t), \lambda \in \mathbb{C}\}$, or equivalently, from $\{\sin (\omega t), \cos (\omega t), \omega \in \mathbb{R}\}$ with $\lambda=i \omega, i^{2}=-1$. The construction of exponentially fitted $\mathrm{RK}(\mathrm{N})$ methods is originally due to Paternoster [19]. After this, the exponentially fitted methods drew a lot of attention. As a result, there are many different types of exponentially fitted RK(N) methods such as $[7,8,12,16,25,26,32]$.

In the last decade, much work related to symmetric, symplectic, and exponentially fitted conditions has emerged. These methods show a better behavior than the other methods which do not possess these three conditions together. The main work focuses on explicit methods for their easy implementation. However, the implicit methods are more suitable for solving stiff ODEs than the explicit methods. There are some researchers working on the implicit RKN methods, such as [13-15, 18]. Especially, in [33], the authors derived an implicit symmetric, symplectic, and exponentially fitted RKN integrator. Exactly, this method is not a true exponential fitting method. For the final stage coefficient $b_{1}$, there are two different expressions. The authors chose $\theta= \pm \frac{\sqrt{3}}{6}$ to make them as close as possible. In this paper, we want to avoid this from happening. So we investigate the modified RungeKutta-Nyström satisfying symmetric, symplectic, and EF conditions. Consequently, we can obtain unique expression of every coefficient which is not true for ISSEFRKN. The new method called ISSEFMRKN also reduces to the classical symplectic, symmetric RKN integrator when the parameter $z$ approaches zero.

The remainder of the paper is organized as follows. We set up symmetric, symplectic, and EF conditions for our modified RKN methods in Sect. 2. In Sect. 3 we derive a class of 
two-stage implicit symmetric symplectic exponentially fitted RKN (EFRKN) integrators. In Sect. 4 we present some numerical experiments that show the accuracy and efficiency of the new method when they are compared with other implicit RKN integrators given in $[1,20,23,24,27,33]$. Finally, Sect. 5 is devoted to some conclusions.

\section{Symmetric, symplectic, exponential fitting conditions}

In this paper, we focus on the following $s$-stage modified implicit RKN method for the second-order ODEs (1):

$$
\left\{\begin{array}{l}
Y_{i}=y_{0}+c_{i} \gamma_{i} h y_{0}^{\prime}+h^{2} \sum_{j=1}^{s} a_{i j} f\left(t_{0}+c_{j} h, Y_{j}\right), \quad i=1, \ldots, s \\
y_{1}=y_{0}+g_{2} h y_{0}^{\prime}+h^{2} \sum_{i=1}^{s} \bar{b}_{i} f\left(t_{0}+c_{i} h, Y_{i}\right), \\
y_{1}^{\prime}=y_{0}^{\prime}+h \sum_{i=1}^{s} b_{i} f\left(t_{0}+c_{i} h, Y_{i}\right) .
\end{array}\right.
$$

This modified RKN method can be expressed by the Butcher tableau

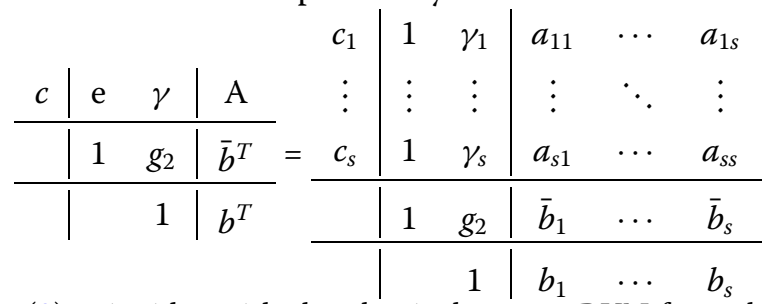

Note that scheme (3) coincides with the classical $s$-stage RKN formula when the coefficients $g_{2}=1, \gamma_{i}=1, i=1, \ldots, s$, and the remaining coefficients are constant. The objective of this section is to find out when the modified RKN scheme (3) is symmetric, symplectic, exponentially fitted respectively. In the following subsections, we put forward these three important properties one by one.

\subsection{Symmetric conditions}

The concept of adjoint method is the hinge of symmetry. First, let us give the definition of adjoint method. We denote a one-step method for second-order ODEs (1) as $\Phi_{h}:\left(y_{0}, y_{0}^{\prime}\right)^{\mathrm{T}} \mapsto\left(y_{1}, y_{1}^{\prime}\right)^{\mathrm{T}}$.

Definition 2.1 The adjoint method $\Phi_{h}^{*}$ of a one-step method $\Phi_{h}$ is the inverse map of the original method with reversed time step $-h$, i.e., $\Phi_{h}^{*}:=\Phi_{-h}^{-1}$. In other words, $y_{1}=\Phi_{h}^{*}\left(y_{0}\right)$ is implicitly defined by $\Phi_{-h}\left(y_{1}\right)=y_{0}$. A method for which $\Phi_{h}^{*}=\Phi_{h}$ is called symmetric (see [11]).

In the case of $s$-stage RKN methods (3), a set of sufficient conditions for the methods to be symmetric are given by

$$
\begin{aligned}
& c_{i}=1-c_{s+1-i}, \quad b_{i}=b_{s+1-i}, \\
& \bar{b}_{i}=g_{2} b_{s+1-i}-\bar{b}_{s+1-i}, \quad c_{i} \gamma_{i}=g_{2}-c_{s+1-i} \gamma_{s+1-i}, \\
& a_{i j}=a_{s+1-i, s+1-j}-c_{s+1-i} \gamma_{s+1-i} b_{s+1-j}+g_{2} b_{s+1-j}-\bar{b}_{s+1-j} .
\end{aligned}
$$

This can be obtained following the procedure in [32] and many other papers. In this paper we consider methods (3) whose coefficients are $z$-dependent, as we do for EF type methods 
$[28,29]$ or adapted type methods $[25,26]$. Then the conditions for the methods to be symmetric are given by

$$
\begin{aligned}
& c_{i}(z)=1-c_{s+1-i}(-z), \quad b_{i}(z)=b_{s+1-i}(-z), \\
& \bar{b}_{i}(z)=g_{2}(-z) b_{s+1-i}(-z)-\bar{b}_{s+1-i}(-z), \\
& c_{i}(z) \gamma_{i}(z)=g_{2}(-z)-c_{s+1-i}(-z) \gamma_{s+1-i}(-z), \\
& a_{i j}(z)=a_{s+1-i, s+1-j}(-z)-c_{s+1-i}(-z) \gamma_{s+1-i}(-z) b_{s+1-j}(-z)+g_{2}(-z) b_{s+1-j}(-z)-\bar{b}_{s+1-j}(-z),
\end{aligned}
$$

where $z=i \omega h, \omega$ is the principal frequency of the problem. We assume that the coefficients of methods (3) are even functions of $h$, as we frequently encounter in the case of EFRKN methods, so that these conditions reduce to (4).

\subsection{Symplectic conditions}

The second important property used in this paper is symplecticity. Now, we turn to the symplectic conditions for scheme (3). Symplecticity is defined for a Hamiltonian system. On many occasions, the problem under consideration takes the form of a Hamiltonian system

$$
\dot{p}=-\frac{\partial}{\partial q} U(t, q), \quad \dot{q}=M^{-1} p,
$$

with the Hamiltonian $H(t, p, q)=\frac{1}{2} p^{\mathrm{T}} M^{-1} p+U(t, q)$, where $M$ is a symmetric positive definite constant matrix. This system is equivalent to the second-order equation (1) with $f(t, q)=-M^{-1} \frac{\partial}{\partial q} U(t, q)$. Now we can give the definition of symplecticity which can be found in [10].

Definition 2.2 A one-step method is symplectic if, for every smooth Hamiltonian function $H$ and for every step size $h$, the corresponding flow preserves the differential 2-form

$$
\mathrm{d} p \wedge \mathrm{d} q=\sum_{i=1}^{d} \mathrm{~d} p_{i} \wedge \mathrm{d} q_{i},
$$

where the one-forms $\mathrm{d} p_{i}$, respectively $\mathrm{d} q_{i}$, map a tangent vector $\xi$ to its $i$ th, respectively $(n+i)$ th, component. Here, we assume that $p$ and $q$ all have $n$ components. Furthermore, $\mathrm{d} p_{i} \wedge \mathrm{d} q_{i}$ is a bilinear map acting on a pair of vectors

$$
\begin{aligned}
\mathrm{d} p_{i} \wedge \mathrm{d} q_{i}\left(\xi_{1}, \xi_{2}\right) & =\operatorname{det}\left(\begin{array}{ll}
\mathrm{d} p_{i}\left(\xi_{1}\right) & \mathrm{d} p_{i}\left(\xi_{2}\right) \\
\mathrm{d} q_{i}\left(\xi_{1}\right) & \mathrm{d} q_{i}\left(\xi_{2}\right)
\end{array}\right) \\
& =\mathrm{d} p_{i}\left(\xi_{1}\right) \mathrm{d} q_{i}\left(\xi_{2}\right)-\mathrm{d} p_{i}\left(\xi_{2}\right) \mathrm{d} q_{i}\left(\xi_{1}\right)
\end{aligned}
$$

and satisfies Grassmann's rules for exterior multiplication

$$
\mathrm{d} p_{i} \wedge \mathrm{d} p_{j}=-\mathrm{d} p_{j} \wedge \mathrm{d} p_{i}, \quad \mathrm{~d} p_{i} \wedge \mathrm{d} p_{i}=0 .
$$

Accordingly, scheme (3) is symplectic if

$$
\mathrm{d} y_{1} \wedge \mathrm{d} y_{1}^{\prime}=\mathrm{d} y_{0} \wedge \mathrm{d} y_{0}^{\prime} .
$$


Using the expressions of $y_{1}$ and $y_{1}^{\prime}$ in (3), we have

$$
\begin{aligned}
\mathrm{d} y_{1} \wedge \mathrm{d} y_{1}^{\prime}= & \mathrm{d} y_{0} \wedge \mathrm{d} y_{0}^{\prime}+h \sum_{i=1}^{s} b_{i} \mathrm{~d} y_{0} \wedge \mathrm{d} f\left(Y_{i}\right)+h g_{2} \mathrm{~d} y_{0}^{\prime} \wedge \mathrm{d} y_{0}^{\prime}+h^{2} \sum_{i=1}^{s} g_{2} b_{i} \mathrm{~d} y_{0}^{\prime} \wedge \mathrm{d} f\left(Y_{i}\right) \\
& +h^{2} \sum_{i=1}^{s} \bar{b}_{i} \mathrm{~d} f\left(Y_{i}\right) \wedge \mathrm{d} y_{0}^{\prime}+h^{3} \sum_{i, j=1}^{s} \bar{b}_{j} b_{i} \mathrm{~d} f\left(Y_{j}\right) \wedge \mathrm{d} f\left(Y_{i}\right)
\end{aligned}
$$

Eliminating $\mathrm{d} y_{0}$ in the second term by inserting the first equation in (3), we obtain

$$
\begin{aligned}
\mathrm{d} y_{1} \wedge \mathrm{d} y_{1}^{\prime}= & \mathrm{d} y_{0} \wedge \mathrm{d} y_{0}^{\prime}+h^{2} \sum_{i=1}^{s}\left(g_{2} b_{i}-b_{i} c_{i} \gamma_{i}-\bar{b}_{i}\right) \mathrm{d} y_{0}^{\prime} \wedge \mathrm{d} f\left(Y_{i}\right) \\
& +\frac{1}{2} h^{3} \sum_{i, j=1}^{s}\left(\bar{b}_{j} b_{i}-b_{i} a_{i j}\right) \mathrm{d} f\left(Y_{j}\right) \wedge \mathrm{d} f\left(Y_{i}\right) \\
& +\frac{1}{2} h^{3} \sum_{i, j=1}^{s}\left(\bar{b}_{i} b_{j}-b_{j} a_{j i}\right) \mathrm{d} f\left(Y_{i}\right) \wedge \mathrm{d} f\left(Y_{j}\right) .
\end{aligned}
$$

Therefore, (3) is symplectic if the following conditions are satisfied:

$$
\begin{aligned}
& \bar{b}_{i}+\left(c_{i} \gamma_{i}-g_{2}\right) b_{i}=0, \quad i=1, \ldots, s, \\
& b_{i}\left(\bar{b}_{j}-a_{i j}\right)=b_{j}\left(\bar{b}_{i}-a_{j i}\right), \quad i, j=1, \ldots, s .
\end{aligned}
$$

\subsection{Exponential fitting conditions}

Following Albrecht's approach [2, 3], each stage of scheme (3) can be viewed as a linear multistep method on a non-equidistant grid. With each stage one can associate a linear function as follows:

- for the internal stages,

$$
\varphi_{i}[y(t) ; h ; \mathbf{a}]=y\left(t+c_{i} h\right)-y(t)-c_{i} \gamma_{i} h y^{\prime}(t)-h^{2} \sum_{j=1}^{s} a_{i j} y^{\prime \prime}\left(t+c_{j} h\right), \quad i=1,2, \ldots, s
$$

- for the final stages,

$$
\begin{aligned}
& \varphi[y(t) ; h ; \overline{\mathbf{b}}]=y(t+h)-y(t)-h g_{2} y^{\prime}(t)-h^{2} \sum_{i=1}^{s} \bar{b}_{i} y^{\prime \prime}\left(t+c_{i} h\right), \\
& \varphi[y(x) ; h ; \mathbf{b}]=y^{\prime}(t+h)-y^{\prime}(t)-h \sum_{i=1}^{s} b_{i} y^{\prime \prime}\left(t+c_{i} h\right) .
\end{aligned}
$$

The following equations can be obtained by requiring that these functions vanish for the functions from the set $\{\exp ( \pm \lambda t) \mid \lambda \in \mathbb{R}$ or $\lambda \in i \mathbb{R}\}$ (here $i$ is the imaginary unit)

$$
\left\{\begin{array}{l}
\left.e^{ \pm c_{i} z}=1 \pm c_{i} \gamma_{i}(z) z+z^{2} \sum_{j=1}^{s} a_{i j}(z) e^{ \pm c_{j} z} \quad \text { (for internal stage } \overline{\mathbf{a}}\right) \\
\left.e^{ \pm z}=1 \pm g_{2} z+z^{2} \sum_{i=1}^{s} \bar{b}_{i}(z) e^{ \pm c_{i} z} \quad \text { (for the final stage } \overline{\mathbf{b}}\right) \\
\left.e^{ \pm z}=1 \pm z \sum_{i=1}^{s} b_{i}(z) e^{ \pm c_{i} z}, \quad z=\lambda h \text { (for the final stage } \mathbf{b}\right)
\end{array}\right.
$$


By definitions of $\cosh (z)=\left(e^{z}+e^{-z}\right) / 2$ and $\sinh (z)=\left(e^{z}-e^{-z}\right) / 2$, equation (6) implies that

$$
\begin{aligned}
& \left\{\begin{array}{l}
\sum_{j=1}^{s} a_{i j}(z) \cosh \left(c_{j} z\right)=\frac{\cosh \left(c_{i} z\right)-1}{z^{2}}, \\
\sum_{j=1}^{s} a_{i j}(z) \sinh \left(c_{j} z\right)=\frac{\sinh \left(c_{i} z\right)-c_{i} \gamma_{i}(z) z}{z^{2}}, \quad i=1, \ldots, s
\end{array}\right. \\
& \begin{cases}\sum_{i=1}^{s} \bar{b}_{i}(z) \cosh \left(c_{i} z\right)=\frac{\cosh (z)-1}{z^{2}}, & \sum_{i=1}^{s} \bar{b}_{i}(z) \sinh \left(c_{i} z\right)=\frac{\sinh (z)-g_{2} z}{z^{2}} \\
\sum_{i=1}^{s} b_{i}(z) \sinh \left(c_{i} z\right)=\frac{\cosh (z)-1}{z}, & \sum_{i=1}^{s} b_{i}(z) \cosh \left(c_{i} z\right)=\frac{\sinh (z)}{z}\end{cases}
\end{aligned}
$$

Equations (7) and (8) are the exponentially fitted conditions for scheme (3). Until now, we have obtained the three pivotal properties used in our method in Sect. 3.

\subsection{Algebraic order conditions}

In this subsection, we will present the algebraic order conditions for exponentially fitted Runge-Kutta-Nystöm (EFRKN) methods. As it occurs in the case of a classical RKN method, for an EFRKN method the local truncation errors in the approximations of the solution and its derivative can be expressed as

$$
\begin{aligned}
& e_{n+1}=y\left(t_{0}+h\right)-y_{1}=\sum_{j=1}^{p-1} h^{j+1}\left(\sum_{i=1}^{k_{j}} d_{i}^{(j+1)} F^{(j)}\left(y_{0}\right)\right)+O\left(h^{p+1}\right), \\
& e_{n+1}^{\prime}=y^{\prime}\left(t_{0}+h\right)-y_{1}^{\prime}=\sum_{j=1}^{p} h^{j}\left(\sum_{i=1}^{k_{j}} d_{i}^{(j)} F^{(j)}\left(y_{0}\right)\right)+O\left(h^{p+1}\right),
\end{aligned}
$$

where $F^{(j)}\left(y_{0}\right)$ denotes an elementary differential and the terms $d_{i}^{(j+1)}$ and ${d^{\prime}}_{i}^{(j)}$ depend on the coefficients of the EFRKN method. Method (3) is of order $p$ if, for every sufficiently smooth IVP(1) and for every small step size $h$, the local truncation errors of the numerical solutions satisfy

$$
\begin{aligned}
& e_{1}=y\left(t_{0}+h\right)-y_{1}=O\left(h^{p+1}\right), \\
& e_{1}^{\prime}=y^{\prime}\left(t_{0}+h\right)-y_{1}^{\prime}=O\left(h^{p+1}\right),
\end{aligned}
$$

or equivalently,

$$
\begin{aligned}
& d_{i}^{(j+1)}=0, \quad i=1, \ldots, k_{j}, j=1, \ldots, p-1, \\
& d_{i}^{\prime(j)}=0, \quad i=1, \ldots, k_{j}, j=1, \ldots, p .
\end{aligned}
$$

In order to obtain the order conditions, we assume the following expansions:

$$
\begin{array}{ll}
\bar{b}_{i}(z)=\bar{b}_{i}^{(0)}+\bar{b}_{i}^{(2)} z^{2}+\bar{b}_{i}^{(4)} z^{4}+\cdots, & b_{i}(z)=b_{i}^{(0)}+b_{i}^{(2)} z^{2}+b_{i}^{(4)} z^{4}+\cdots, \\
\gamma_{i}(z)=1+\gamma_{i}^{(2)} z^{2}+\gamma_{i}^{(4)} z^{4}+\cdots, & a_{i j}(z)=a_{i j}^{(0)} a_{i j}^{(2)} z^{2}+a_{i j}^{(4)} z^{4}+\cdots, \\
g_{2}(z)=1+g_{2}^{(2)} z^{2}+g_{2}^{(4)} z^{4}+\cdots . &
\end{array}
$$

Then the order conditions up to fourth order for (3) are presented as follows. 
Order 1 requires:

$$
d_{1}^{(1)}:=\sum_{i} b_{i}^{(0)}-1=0 .
$$

Order 2 requires in addition:

$$
d_{1}^{\prime(2)}:=\sum_{i} b_{i}^{(0)} c_{i}-\frac{1}{2}=0, \quad d_{1}^{(2)}:=\sum_{i} \bar{b}_{i}^{(0)}-\frac{1}{2}=0 .
$$

Order 3 requires in addition:

$$
\begin{aligned}
& d_{1}^{\prime(3)}:=\frac{1}{2}\left(\sum_{i} b_{i}^{(0)} c_{i}^{2}-\frac{1}{3}\right)=0, \quad d_{2}^{\prime(3)}:=\sum_{i} b_{i}^{(0)} \sum_{k} a_{i k}^{(0)}-\frac{1}{6}=0, \\
& d_{3}^{\prime(3)}:=\sum_{i} b_{i}^{(2)}=0, \quad d_{1}^{(3)}:=\sum_{i} \bar{b}_{i}^{(0)} c_{i}-\frac{1}{6}=0, \\
& d_{2}^{(3)}:=g_{2}^{(2)}=0 .
\end{aligned}
$$

Order 4 requires in addition:

$$
\begin{aligned}
& d_{1}^{(4)}:=\frac{1}{6}\left(\sum_{i} b_{i}^{(0)} c_{i}^{3}-\frac{1}{4}\right)=0, \quad d_{2}^{(4)}:=\sum_{i} b_{i}^{(0)} \sum_{k} c_{i} a_{i k}^{(0)}-\frac{1}{8}=0 \\
& d_{3}^{(3)}:=\sum_{i} b_{i}^{(0)} \sum_{k} a_{i k}^{(0)} c_{k}-\frac{1}{24}=0, \quad d_{4}^{(4)}:=\sum_{i} b_{i}^{(0)} c_{i} \gamma_{i}^{(2)}=0 \\
& d_{5}^{(4)}:=\sum_{i} b_{i}^{(2)} c_{i}=0, \quad d_{1}^{(4)}:=\frac{1}{2}\left(\sum_{i} \bar{b}_{i}^{(0)} c_{i}^{2}-\frac{1}{12}\right)=0 \\
& d_{2}^{(4)}:=\sum_{i} \bar{b}_{i}^{(0)} \sum_{k} a_{i k}^{(0)}-\frac{1}{24}=0, \quad d_{3}^{(4)}:=\sum_{i} \bar{b}_{i}^{(2)}=0
\end{aligned}
$$

From Theorem 2.1 in [8], we know that the modified RKN method (3) has algebraic order at least 2 .

\section{Construction of implicit symmetric and symplectic EFRKN methods}

In this section we construct an implicit EFRKN method under the symmetry, symplecticity, exponential fitting conditions obtained in the previous section.

In this paper, we consider two simple cases: $s=1$ and $s=2$.

- $s=1$

When $s=1$, the symmetry conditions (4) and the symplecticity conditions (5) reduce to

$$
c_{1}=1 / 2, \quad \bar{b}_{1}=g_{2} b_{1} / 2, \quad g_{2}=\gamma_{1} .
$$

When $s=1$, the exponentially fitted conditions (7) and (8) can be written as

$$
\left\{\begin{array}{lc}
a_{11} \cosh \left(c_{1} z\right)=\frac{\cosh \left(c_{1} z\right)-1}{z^{2}}, & a_{11} \sinh \left(c_{1} z\right)=\frac{\sinh \left(c_{1} z\right)-c_{1} \gamma_{1} z}{z^{2}}, \\
\bar{b}_{1} \cosh \left(c_{1} z\right)=\frac{\cosh (z)-1}{z^{2}}, & \bar{b}_{1} \sinh \left(c_{1} z\right)=\frac{\sinh (z)-g_{2} z}{z^{2}} \\
b_{1} \cosh \left(c_{1} z\right)=\frac{\sinh (z)}{z}, & b_{1} \sinh \left(c_{1} z\right)=\frac{\cosh (z)-1}{z}
\end{array}\right.
$$


Consider $c_{1}=1 / 2$ in (10), we have

$$
\begin{cases}a_{11}=\frac{\cosh (z / 2)-1}{z^{2} \cosh (z / 2)}, & \gamma_{1}=\frac{2 \sinh (z / 2)}{z \cosh (z / 2)} \\ \bar{b}_{1}=\frac{\cosh (z)-1}{z^{2} \cosh (z / 2)}, & g_{2}=\frac{2 \sinh (z / 2)}{z \cosh (z / 2)} \\ b_{1}=\frac{2 \sinh (z / 2)}{z} & \end{cases}
$$

Obviously, the third equation in (9) is satisfied, i.e., $g_{2}=\gamma_{1}$. By some simple calculation, it is easy to verify that $\bar{b}_{1}=g_{2} b_{1} / 2$. Thus, we obtain all the coefficients as follows:

$$
\left\{\begin{array}{l}
c_{1}=1 / 2, \quad a_{11}=\frac{\cosh (z / 2)-1}{z^{2} \cosh (z / 2)}, \quad g_{2}=\gamma_{1}=\frac{2 \sinh (z / 2)}{z \cosh (z / 2)}, \\
\bar{b}_{1}=\frac{\cosh (z)-1}{z^{2} \cosh (z / 2)}, \quad b_{1}=\frac{2 \sinh (z / 2)}{z}
\end{array}\right.
$$

Furthermore, the Taylor expansion of all the coefficients is

$$
\left\{\begin{array}{l}
a_{11}=\frac{1}{8}-\frac{5}{384} z^{2}+\frac{61}{46,080} z^{4}+\cdots, \quad g_{2}=\gamma_{1}=1-\frac{1}{12} z^{2}+\frac{1}{120} z^{4}+\cdots, \\
\bar{b}_{1}=\frac{1}{2}-\frac{1}{48} z^{2}+\frac{31}{11,520} z^{4}+\cdots,
\end{array}\right.
$$

It is easy to verify that all these coefficients satisfy all the second-order conditions, but do not satisfy the third-order condition $d_{1}^{\prime(3)}:=\frac{1}{2}\left(\sum_{i} b_{i}^{(0)} c_{i}^{2}-\frac{1}{3}\right)=0$. Thus this one-stage method is of order 2. We denote it as ISSEFMRKNs1o2, and "s1o2" means stage 1 order 2.

- $s=2$

In this case, the symmetry conditions (4) and the symplecticity conditions (5) reduce to

$$
\begin{aligned}
& c_{1}+c_{2}=1, \quad b_{1}=b_{2}, \quad \bar{b}_{1}+\bar{b}_{2}=g_{2} b_{1}, \quad c_{1} \gamma_{1}+c_{2} \gamma_{2}=g_{2}, \\
& a_{12}=a_{21}+b_{1}\left(c_{1} \gamma_{1}-c_{2} \gamma_{2}\right), \quad a_{21}=a_{12}+b_{2}\left(c_{2} \gamma_{2}-c_{1} \gamma_{1}\right), \\
& \bar{b}_{1}=b_{1} c_{2} \gamma_{2}, \quad \bar{b}_{2}=b_{2} c_{1} \gamma_{1}, \quad b_{1} \bar{b}_{2}-b_{1} a_{12}=b_{2} \bar{b}_{1}-b_{2} a_{21} \text {. }
\end{aligned}
$$

Considering symmetric condition $c_{1}+c_{2}=1$, we introduce an extra parameter $\theta$ and assume $c_{1}=\frac{1}{2}-\theta, c_{2}=\frac{1}{2}+\theta$. Then equation (12) becomes

$$
\begin{array}{ll}
c_{1}=\frac{1}{2}-\theta, \quad c_{2}=\frac{1}{2}+\theta, & b_{2}=b_{1}, \quad \bar{b}_{1}=b_{1} c_{2} \gamma_{2}, \quad \bar{b}_{2}=b_{1} c_{1} \gamma_{1}, \\
a_{21}-a_{12}=b_{1}\left(c_{2} \gamma_{2}-c_{1} \gamma_{1}\right), & g_{2}=c_{1} \gamma_{1}+c_{2} \gamma_{2} .
\end{array}
$$

Now we simplify the exponentially fitted conditions (7) and (8) when $s=2$. By using $\bar{b}_{1}=b_{1} c_{2} \gamma_{2}, \bar{b}_{2}=b_{1} c_{1} \gamma_{1}, a_{21}-a_{12}=b_{1}\left(c_{2} \gamma_{2}-c_{1} \gamma_{1}\right)$, the EF conditions are

$$
\begin{aligned}
& b_{1}\left(c_{1} \gamma_{1}-c_{2} \gamma_{2}\right)=\frac{2 \sinh (z / 2)-g_{2} z \cosh (z / 2)}{z^{2} \sinh (\theta z)}, \\
& b_{1}=\frac{\sinh (z / 2)}{z \cosh (\theta z)}, \\
& \left(a_{11}+a_{12}\right) \cosh (\theta z)=\frac{\cosh (\theta z)-\cosh (z / 2)+c_{1} \gamma_{1} z \sinh (z / 2)}{z^{2}},
\end{aligned}
$$




$$
\begin{aligned}
& \left(a_{12}-a_{11}\right) \sinh (\theta z)=\frac{\sinh (z / 2)-\sinh (\theta z)-c_{1} \gamma_{1} z \cosh (z / 2)}{z^{2}} \\
& \left(a_{21}+a_{22}\right) \cosh (\theta z)=\frac{\cosh (\theta z)-\cosh (z / 2)+c_{2} \gamma_{2} z \sinh (z / 2)}{z^{2}} \\
& \left(a_{22}-a_{21}\right) \sinh (\theta z)=\frac{\sinh (z / 2)+\sinh (\theta z)-c_{2} \gamma_{2} z \cosh (z / 2)}{z^{2}}
\end{aligned}
$$

From these conditions, we cannot determine the specific expressions of all the coefficients. So we impose that the final stage is also exact for the functions $\{\exp (2 \lambda t), \exp (-2 \lambda t)\}$, i.e., the following conditions are also satisfied:

$$
\begin{cases}\sum_{i=1}^{s} \bar{b}_{i}(z) \cosh \left(2 c_{i} z\right)=\frac{\cosh (2 z)-1}{4 z^{2}}, & \sum_{i=1}^{s} \bar{b}_{i}(z) \sinh \left(2 c_{i} z\right)=\frac{\sinh (2 z)-2 g_{2} z}{4 z^{2}} \\ \sum_{i=1}^{s} b_{i}(z) \sinh \left(2 c_{i} z\right)=\frac{\cosh (2 z)-1}{2 z}, & \sum_{i=1}^{s} b_{i}(z) \cosh \left(2 c_{i} z\right)=\frac{\sinh (2 z)}{2 z}\end{cases}
$$

Inserting $c_{1}=\frac{1}{2}-\theta, c_{2}=\frac{1}{2}+\theta, b_{1}=b_{2}, \bar{b}_{1}=b_{1} c_{2} \gamma_{2}, \bar{b}_{2}=b_{1} c_{1} \gamma_{1}$ into (20), and after some simple calculations, we have

$$
\begin{aligned}
& b_{1}\left(c_{1} \gamma_{1}-c_{2} \gamma_{2}\right)=\frac{\sinh (z)-g_{2} z \cosh (z)}{2 z^{2} \sinh (2 \theta z)}, \\
& b_{1}=\frac{\sinh (z)}{2 z \cosh (2 \theta z)} .
\end{aligned}
$$

Combining (15) and (22), we obtain

$$
b_{1}=\frac{\sinh (z / 2)}{z \cosh (\theta z)}, \quad \theta=\frac{\operatorname{arccosh}(\beta)}{z}, \quad \beta=\frac{\cosh (z / 2)+\sqrt{8+\cosh ^{2}(z / 2)}}{4} .
$$

In order to determine the RKN methods when $z \rightarrow 0$, we present the Taylor expansion of $\theta$, i.e.,

$$
\theta=\frac{\sqrt{3}}{6}\left(1+\frac{1}{72} z^{2}+\cdots\right)
$$

Using $c_{1} \gamma_{1}+c_{2} \gamma_{2}=g_{2},(14)$, and (21), we obtain

$$
\left\{\begin{array}{l}
g_{2}=\frac{4 \sinh (z / 2) \sinh (2 \theta z)-\sinh (z) \sinh (\theta z)}{z(2 \cosh (z / 2) \sinh (2 \theta z)-\cosh (z) \sinh (\theta z))} \\
\gamma_{1}=\frac{2 \sinh (z / 2)-g_{2} z \cosh (z / 2)}{2 c_{1} z^{2} \sinh (\theta z) b_{1}}+\frac{g_{2}}{2 c_{1}}
\end{array}\right.
$$

Furthermore, if we assume $a_{11}=a_{22}$, we can find that (16) - (18) is equivalent to (15) and $(17)+(19)$ is equivalent to (14). So, method (3) satisfying (14)-(17) is exponentially fitted. From equations (16) and (17), we have

$$
a_{11}=\frac{\sinh (2 \theta z)-\sinh \left(c_{2} z\right)+c_{1} \gamma_{1} z \cosh \left(c_{2} z\right)}{z^{2} \sinh (2 \theta z)}, \quad a_{12}=\frac{\sinh \left(c_{1} z\right)-c_{1} \gamma_{1} z \cosh \left(c_{1} z\right)}{z^{2} \sinh (2 \theta z)}
$$


Until now, we have obtained an implicit symmetric and symplectic exponentially fitted Runge-Kutta-Nyström method whose coefficients are given as

$$
\left\{\begin{array}{l}
\theta=\frac{\operatorname{arccosh}(\beta)}{z}, \quad \beta=\frac{\cosh (z / 2)+\sqrt{8+\cosh ^{2}(z / 2)}}{4}, \\
c_{1}=\frac{1}{2}-\theta, \quad c_{2}=\frac{1}{2}+\theta, \quad g_{2}=\frac{4 \sinh (z / 2) \sinh (2 \theta z)-\sinh (z) \sinh (\theta z)}{z(2 \cosh (z / 2) \sinh (2 \theta z)-\cosh (z) \sinh (\theta z))}, \\
b_{1}=\frac{\sinh (z / 2)}{z \cosh (\theta z)}, \quad \gamma_{1}=\frac{2 \sinh (z / 2)-g_{2} z \cosh (z / 2)}{2 c_{1} z^{2} \sinh (\theta z) b_{1}}+\frac{g_{2}}{2 c_{1}}, \\
\gamma_{2}=\frac{g_{2}-c_{1} \gamma_{1}}{c_{2}}, \quad b_{2}=b_{1}, \quad \bar{b}_{1}=b_{1}\left(g_{2}-c_{1} \gamma_{1}\right), \quad \bar{b}_{2}=b_{1} c_{1} \gamma_{1} \\
a_{11}=\frac{\sinh (2 \theta z)-\sinh \left(c_{2} z\right)+c_{1} \gamma_{1} z \cosh \left(c_{2} z\right)}{z^{2} \sinh (2 \theta z)}, \quad a_{22}=a_{11}, \\
a_{12}=\frac{\sinh \left(c_{1} z\right)-c_{1} \gamma_{1} z \cosh \left(c_{1} z\right)}{z^{2} \sinh (2 \theta z)}, \quad a_{21}=a_{12}+b_{1}\left(g_{2}-2 c_{1} \gamma_{1}\right) .
\end{array}\right.
$$

We denote this method as ISSEFMRKNs2o4, and "s2o4" means stage 2 order 4. For small values of $z$, the Taylor series expansions of the coefficients are given by

$$
\left\{\begin{array}{l}
\gamma_{1}=1-\frac{1+\sqrt{3}}{72} z^{2}+\cdots, \quad \gamma_{2}=1-\frac{1-\sqrt{3}}{72} z^{2}+\cdots, \quad g_{2}=1+\frac{1}{270} z^{4}+\cdots, \\
a_{11}=\frac{1}{72}-\frac{119}{51,840} z^{2}+\cdots, \quad a_{12}=\frac{11-6 \sqrt{3}}{72}+\frac{239-180 \sqrt{3}}{51,840} z^{2}+\cdots \\
a_{21}=\frac{11+6 \sqrt{3}}{72}+\frac{239+180 \sqrt{3}}{51,840} z^{2}+\cdots, \quad b_{1}=\frac{1}{2}-\frac{z^{4}}{2160}+\cdots \\
\bar{b}_{1}=\frac{3+\sqrt{3}}{12}+\frac{\sqrt{3}}{288} z^{2}+\cdots, \quad \bar{b}_{2}=\frac{3-\sqrt{3}}{12}-\frac{\sqrt{3}}{288} z^{2}+\cdots
\end{array}\right.
$$

From these Taylor series, we can verify that our method ISSEFMRKNs2o4 satisfies all the fourth-order conditions. So, the method ISSEFMRKNs2o4 is of order 4. The method ISSEFRKNs2o4 proposed in [33] is of order four with $\theta= \pm \frac{\sqrt{3}}{6}$. Thus the nodes are constant. However, in our method ISSEFMRKNs2o4, $\theta=\frac{\operatorname{arccosh}(\beta)}{z}$ which is a function of $z$. That makes the nodes here variables. But when $z \rightarrow 0$, ISSEFMRKNs2o4 and ISSEFRKN2 reduce to the symmetric and symplectic method proposed in [20]. In detail, ISSEFRKNs2o4 proposed in [33] reduces to SSRKN of order 4 in [20] with $a_{11}=\frac{1}{36}$, ISSEFMRKNs2o4 derived in this paper reduces to SSRKN of order 4 in [20] with $a_{11}=\frac{1}{72}$.

\section{Numerical experiments}

In this section, we are dedicated to some numerical studies to illustrate the effectiveness and efficiency of the derived methods ISSEFMRKNs1o2 and ISSEFMRKNs2o4. We select four problems which are often used in many literature works to accomplish the numerical experiments. We also use some other methods for comparisons:

- DIRKN4(3): The embedded diagonally implicit RKN 4(3) pair method proposed by Al-Khasawneh et al. in [1].

- DIRKNs3o4: The diagonally implicit three-stage RKN of order four proposed by Senu et al. in [23].

- ISSRKNs2o4: The implicit symmetric and symplectic two-stage RKN of order four proposed by Qin et al. in [20] with $a_{11}=\frac{1}{72}$.

- ISSEFRKN2: The implicit symmetric and symplectic exponentially fitted two-stage RKN of order four proposed in [33].

- ISSEFMRKNs1o2: The implicit symmetric and symplectic exponentially fitted one-stage modified RKN (11) of order two proposed in this paper.

- ISSEFMRKNs2o4: The implicit symmetric and symplectic exponentially fitted two-stage modified RKN (23) of order four proposed in this paper. 


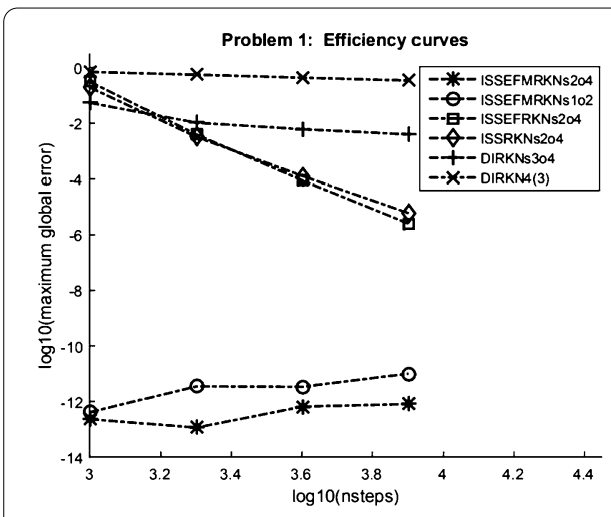

(a)

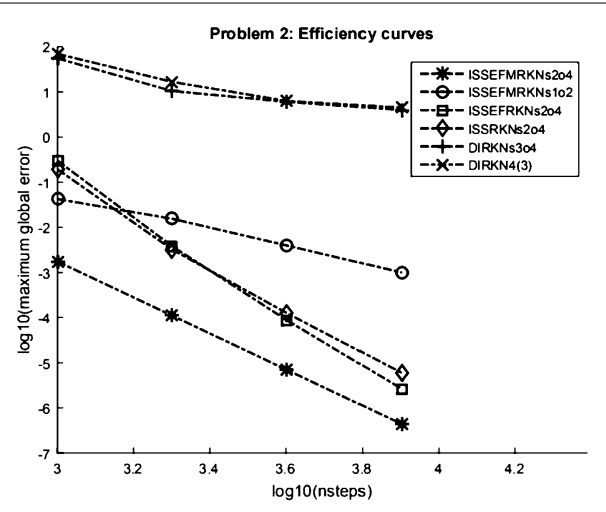

(b)

Figure 1 Efficiency curves for solution in (a) Problem 1, (b) Problem 2

In our numerical experiments, the following nonlinear equations need to be solved:

$$
\left\{\begin{array}{l}
Y_{1}=y_{0}+c_{1} \gamma_{1} h y_{0}^{\prime}+h^{2}\left(a_{11} f\left(t_{0}+c_{1} h, Y_{1}\right)+a_{12} f\left(t_{0}+c_{2} h, Y_{2}\right)\right) \\
Y_{2}=y_{0}+c_{2} \gamma_{2} h y_{0}^{\prime}+h^{2}\left(a_{21} f\left(t_{0}+c_{1} h, Y_{1}\right)+a_{22} f\left(t_{0}+c_{2} h, Y_{2}\right)\right) .
\end{array}\right.
$$

In our algorithm, we use the Newton iteration method to accomplish this process. To be specific, the initial values are taken as $Y_{1}^{(0)}=Y_{2}^{(0)}=y(0)$. Once the difference between the Euclidean norm of two successive iterations exceeds $10^{-8}$, the iteration will be stopped. And we also end the iteration when the number of iterations is more than 1000.

The measurement used in the numerical comparisons is the usual test based on computing the maximum global error in the solution over the whole integration interval. In Figs. 1-3 we show the decimal logarithm of the maximum global error $(\log 10$ (maximum global error)) versus the number of steps required by each code on a logarithmic scale $(\log 10$ (nsteps)). We also integrate the problem on a long time interval with a fixed step size and plot the time evolution of the maximal global error of the Hamiltonian. We have implemented all the codes in MATLAB (Version R2015b). All runs are performed on a laptop with Intel Core(TM)i7-2640M CPU (2.80 GHz) and 8 GB RAM. And all computations are carried out in double precision arithmetic (16 significant digits of accuracy).

Problem 1 We consider the perturbed orbital problem (studied in [8])

$$
\begin{cases}q_{1}^{\prime \prime}=-\frac{q_{1}}{\left(q_{1}^{2}+q_{2}^{2}\right)^{3 / 2}}-\frac{\left(2 \varepsilon+\varepsilon^{2}\right) q_{1}}{\left(q_{1}^{2}+q_{2}^{2}\right)^{5 / 2}}, & t \in\left[0, t_{\mathrm{end}}\right], \\ q_{2}^{\prime \prime}=-\frac{q_{2}}{\left(q_{1}^{2}+q_{2}^{2}\right)^{3 / 2}}-\frac{\left(2 \varepsilon \varepsilon^{2}\right) q_{2}}{\left(q_{1}^{2}+q_{2}^{2}\right)^{5 / 2}}, & \\ q_{1}(0)=1, \quad q_{1}^{\prime}(0)=0, & q_{2}(0)=0, \quad q_{2}^{\prime}(0)=1+\varepsilon .\end{cases}
$$

The Hamiltonian of this problem is given by

$$
H(p, q)=\frac{1}{2}\left(p_{1}^{2}+p_{2}^{2}\right)-\frac{1}{\left(q_{1}^{2}+q_{2}^{2}\right)^{1 / 2}}-\frac{2 \varepsilon+\varepsilon^{2}}{3\left(q_{1}^{2}+q_{2}^{2}\right)^{3 / 2}},
$$




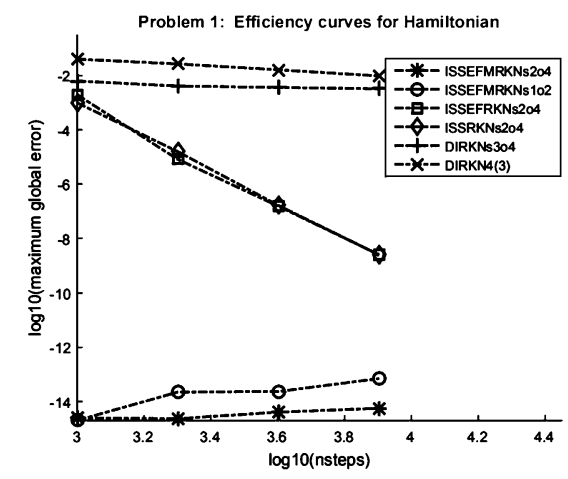

(a)

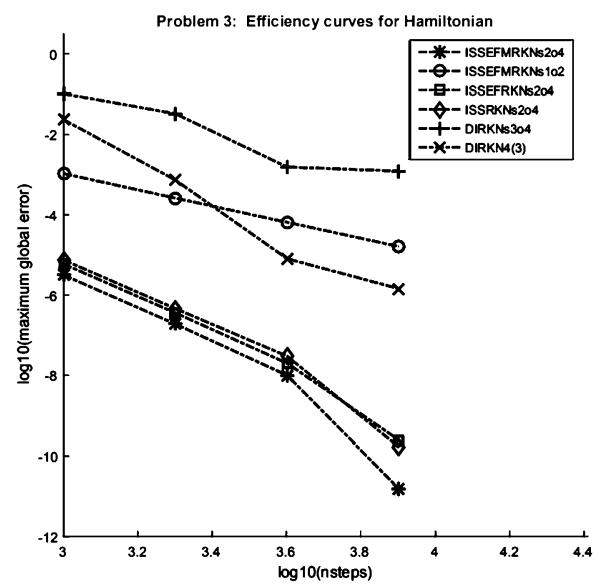

(c)

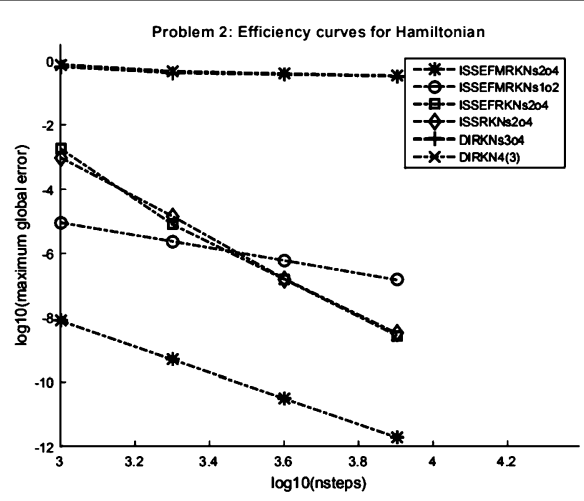

(b)

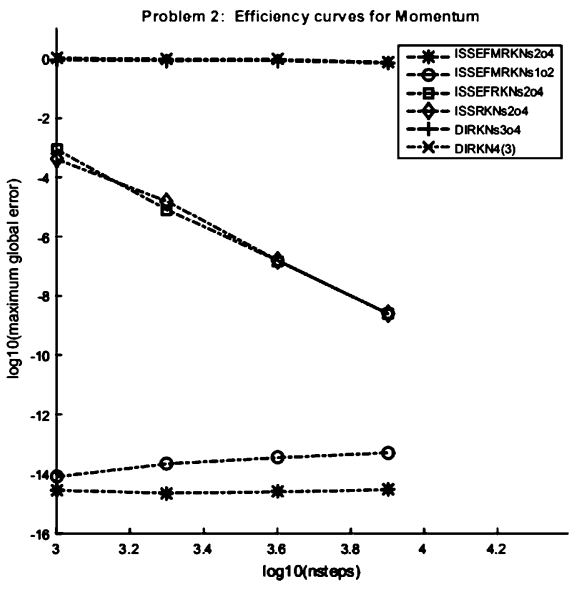

(d)

Figure 2 Efficiency curve for Hamiltonian in (a) Problem 1, (b) Problem 2, (c) Problem 3 and for momentum accuracy comparison in (d) Problem 2

where $\left(p_{1}, p_{2}\right)=\left(q_{1}^{\prime}, q_{2}^{\prime}\right)$. The exact solution is

$$
q_{1}(t)=\cos ((1+\varepsilon) t), \quad q_{2}(t)=\sin ((1+\varepsilon) t)
$$

In this paper, we consider a special case $\varepsilon=0.001$. In our numerical study, we choose $\omega=1+\varepsilon, t_{\text {end }}=500$, and $h=1 / 2^{m}, m=1,2,3$, . Figure 1 (a) shows the efficient curve of the solution. And we presented the efficient curve for Hamiltonian Fig. 2(a).

Problem 2 The two-body problem (studied in [11]) consists of finding the positions and velocities of two massive bodies that attract each other gravitationally, given their masses, positions, and velocities at some initial time. The first body is located in the origin, while the second body is located in the plane with Cartesian coordinates $\left(q_{1}, q_{2}\right)$. The velocity of the second body is given by $\left(q_{1}^{\prime}, q_{2}^{\prime}\right)=\left(p_{1}, p_{2}\right)$. The Hamiltonian is given by

$$
H(p, q)=\frac{1}{2}\left(p_{1}^{2}+p_{2}^{2}\right)-\frac{1}{\sqrt{q_{1}^{2}+q_{2}^{2}}} .
$$




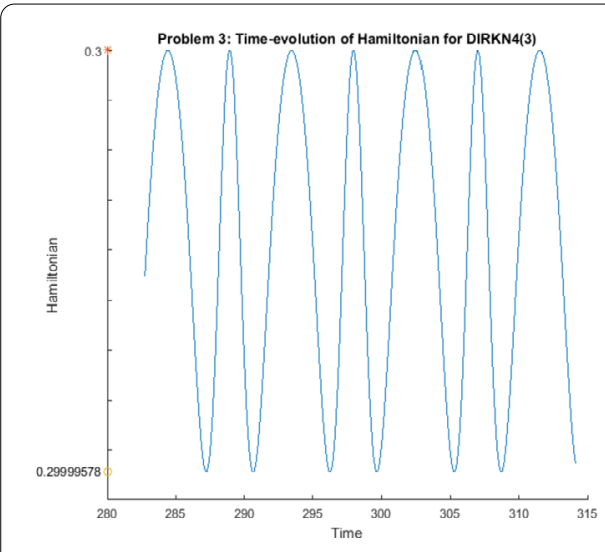

(a)

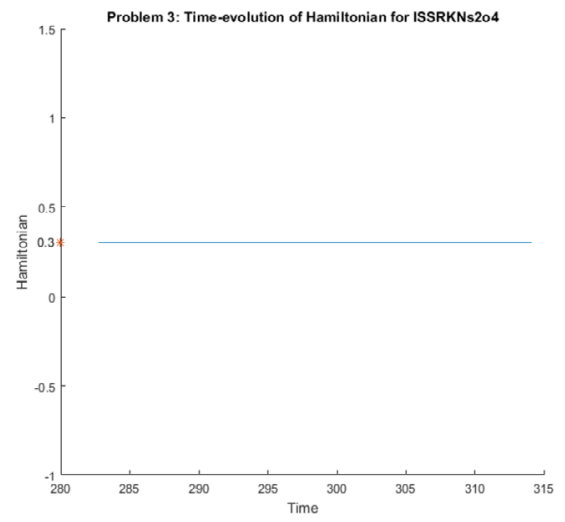

(c)

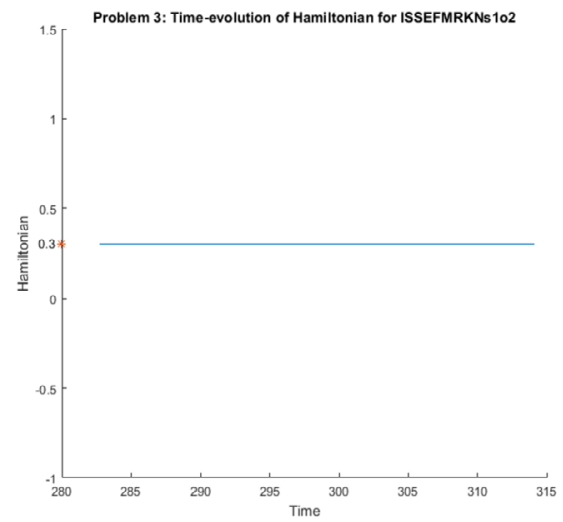

(e)

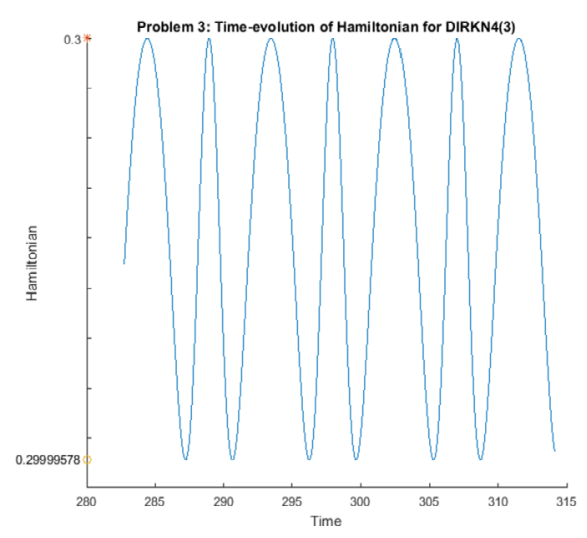

(b)

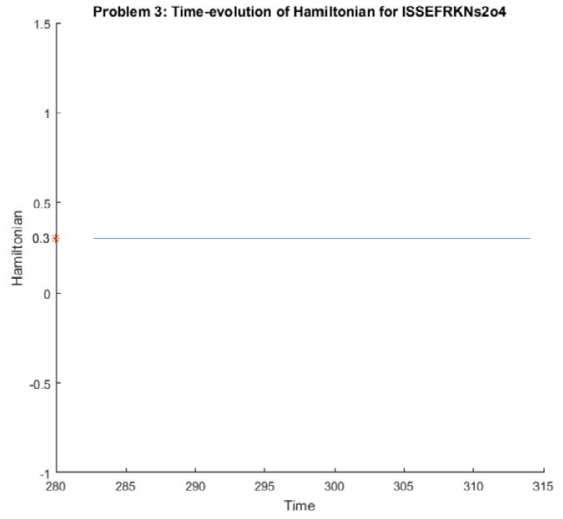

(d)

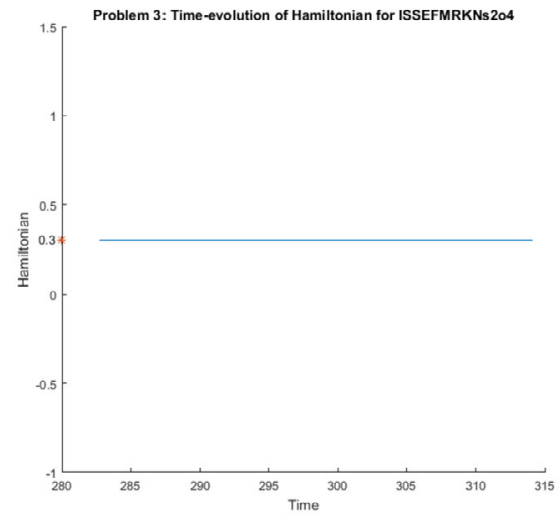

(f)

Figure 3 Numerical Hamiltonian for the methods (a) DIRKN4(3), (b) DIRKNs304, (c) ISSRKNs204, (d) ISSEFRKNs204, (e) ISSEFMRKNs102, and (f) ISSEFMRKNs204 in Problem 3

The Hamiltonian system is equivalent to the following second-order problem:

$$
\left\{\begin{array}{l}
q_{1}^{\prime \prime}=-\frac{q_{1}}{\left(q_{1}^{2}+q_{2}^{2}\right)^{3 / 2}}, \\
q_{2}^{\prime \prime}=-\frac{q_{2}}{\left(q_{1}^{2}+q_{2}^{2}\right)^{3 / 2}} .
\end{array}\right.
$$


The initial values are taken as $q_{1}(0)=1-e, q_{1}^{\prime}(0)=0, q_{2}(0)=0, q_{2}^{\prime}(0)=\sqrt{\frac{1+e}{1-e}}$ with $0<e<1$. The exact solution of the problem is

$$
\left\{\begin{array}{l}
q_{1}(t)=\cos (E)-e \\
q_{2}(t)=\sqrt{1-e^{2}} \sin (E),
\end{array}\right.
$$

where $e$ is the eccentricity of the orbit and the eccentric anomaly $E$ is expressed as an implicit function of the independent variable $t$ by Kepler's equation $t=E-e \sin (E)$. The system has the conservative energy $H$ and the conservative angular momentum $M=q_{1} p_{2}-q_{2} p_{1}$. The initial energy $H_{0}=-\frac{1}{2}$ and the initial momentum $M_{0}=\sqrt{1-e^{2}}$.

In this experiment, we choose $e=10^{-2}$. Figure 2(b) and (d) present the efficiency curves for the Hamiltonian and for the momentum, respectively, on the interval $[0,500]$ with the step sizes $h=1 / 2^{m}, m=1, \ldots, 4$.

Problem 3 Consider the pendulum problem with the Hamiltonian (studied in [22])

$$
H(p, q)=\frac{1}{2} p^{2}-a \cos (q), \quad a>0 .
$$

The Hamiltonian system is equivalent to the following second-order equation:

$$
q^{\prime \prime}=-a \sin (q)
$$

The initial values are $q(0)=0, q^{\prime}(0)=1$. For these initial conditions the constant value of the Hamiltonian is $H_{0}=1 / 2-a$. Since for $|q|<1$ the equation can be expanded as $q^{\prime \prime}+a q=a\left(\frac{q^{3}}{3 !}-\frac{q^{5}}{5 !}+\cdots\right)$, a reasonable choice for the fitted frequency is $\omega=\sqrt{a}$. We take the parameter $a=0.2$. First, we set the interval $[0,500]$ with the step sizes $h=1 / 2^{m}, m=$ $1, \ldots, 4$, to show the accuracy of Hamiltonian in Fig. 2(c). Then the problem is integrated on the interval $[0,100 \pi]$ and the step size $h=1 / 10 \pi$. Figure 3 presents the time evolution of numerical Hamiltonian for each method on the interval $[90 \pi, 100 \pi]$.

From Figs. 1-3, we can find that the implicit modified EFRKN method ISSEFMRKNs2o4 is more efficient than other methods used for comparison in this paper. However, sometimes the method ISSEFMRKNs1o2 performs not better than other methods. From Fig. 2 and Fig. 3, we can see that symplectic methods ISSRKNs2o4, ISSEFRKNs2o4, ISSEFMRKNs1o2, and ISSEFMRKNs2o4 show a better behavior than un-symplectic methods DIRKNs3o4 and DIRKN4(3). This illustrates the special property - symplecticity. Especially, for problem 3, symplectic methods preserve the Hamiltonian much more accurately than the methods which are not symplectic. Comparing the modified method ISSEFMRKNs2o4 and the unmodified code ISSEFRKNs2o4 proposed in [33], we find that ISSEFMRKNs2o4 gives a better behavior than ISSEFRKNs2o4, although they have the same order (order 4).

\section{Conclusions}

In this paper, we focus on a class of two-stage IEFMRKN integrators which are symmetric and symplectic and exponentially fitted. In addition, their final stages also preserve the quadratic invariants $\{\exp (2 \lambda t), \exp (-2 \lambda t)\}$. Like some existing EFRKN integrators (see $[17,32,33]$ for example), the coefficients of the new methods depend on the product of the 
dominant frequency $\omega$ and the step size $h$. Especially, we derived two ISSEFMRKN methods: ISSEFMRKNs1o2 and ISSEFMRKNs2o4. They reduce to the classical RKN method as the parameter $\lambda h$ approaches zero. From the numerical experiments, we can find that these two new methods are more efficient than unmodified ISSEFRKN and some other integrators used for comparison. However, the algebraic order of ISSEFMRKNs1o2 and ISSEFMRKNs2o4 are 2, 4, respectively, which is not high. Thus in the future, we will consider the derivation of high order ISSEFRKN methods, such as six order, eight order.

\section{Acknowledgements \\ The authors are grateful to the anonymous referees for their careful reading of the manuscript and for their invaluable} comments and suggestions, which largely helped to improve this paper.

\section{Funding}

The research of Wenjuan Zhai was supported in part by the Project for Youth Scholars of Higher Education of Hebei Province (QN2017402), and the Project of Teaching and Research of Beijing Jiaotong University Haibin College (HBJY16005).

\section{Availability of data and materials}

No applicable.

\section{Competing interests}

The authors declare that there is no conflict of interests regarding the publication of this paper.

\section{Authors' contributions}

$\mathrm{HZ}$ derived the method ISSEFMRKNS102. And he redid all the new numerical experiments. He also refined the English of our manuscript. WZ derived the symmetric conditions, symplectic conditions, and exponentially fitted conditions and gave the method ISSEFMRKNs204. BC wrote the first draft and organized the whole paper. All authors read and approved the final manuscript.

\section{Author details}

${ }^{1}$ School of Economics and Management, Beijing Jiaotong University, Beijing, China. ${ }^{2}$ Department of Mathematics, Beijing Jiaotong University Haibin College, Cangzhou, China. ${ }^{3}$ School of Science, Beijing Jiaotong University, Beijing, China.

\section{Publisher's Note}

Springer Nature remains neutral with regard to jurisdictional claims in published maps and institutional affiliations.

Received: 23 August 2018 Accepted: 2 December 2018 Published online: 13 December 2018

\section{References}

1. Al-Khasawneh, R.A., Ismail, F., Suleiman, M.: Embedded diagonally implicit Runge-Kutta-Nyström 4(3) pair for solving special second-order IVPs. Appl. Math. Comput. 190, 1803-1814 (2007)

2. Albrecht, P.: The extension of the theory of A-methods to RK methods. In: Strehmel, K. (ed.) Numerical Treatment of Differential Equations. Proceedings of the 4th Seminar NUMDIFF-4, Tuebner-Texte Zur Mathematik, pp. 8-18. Tuebner, Leipzig (1987)

3. Albrecht, P.: A new theoretical approach to Runge-Kutta methods. SIAM J. Numer. Anal. 24, 391-406 (1987)

4. Calvo, M.P., Sanz-Serna, J.M.: High-order symplectic Runge-Kutta-Nyström methods. SIAM J. Sci. Comput. 14, 1237-1252 (1993)

5. Coleman, J.P., Ixaru, L.Gr.: P-stability and exponential-fitting methods for $y^{\prime \prime}=f(x, y)$. IMA J. Numer. Anal. 6, 179-199 (1996)

6. Franco, J.M.: Runge-Kutta-Nyström methods adapted to the numerical integration of perturbed oscillators. Comput. Phys. Commun. 147, 770-782 (2002)

7. Franco, J.M.: Exponentially fitted explicit Runge-Kutta-Nyström methods. J. Comput. Appl. Math. 167, 1-19 (2004)

8. Franco, J.M.: Exponentially fitted symplectic integrators of RKN type for solving oscillatory problems. Comput. Phys. Commun. 177, 479-492 (2007)

9. Franco, J.M., Gomez, l.: Symplectic explicit methods of Runge-Kutta-Nyström type for solving perturbed oscillators J. Comput. Appl. Math. 260, 482-493 (2014)

10. Hairer, E., Lubich, C., Wanner, G.: Geometric Numerical Integration: Structure Preserving Algorithms for Ordinary Differential Equations. Springer, Berlin (2002)

11. Hairer, E., Nørsett, S.P., Wanner, G.: Solving Ordinary Differential Equations I, Nonstiff Problems, Second revised edn. Springer, Berlin (1993)

12. Ixaru, Gr.L., Vanden Berghe, G.: Exponential Fitting. Kluwer Academic Publishers, Dordrecht (2004)

13. Jator, S.N.: Implicit third derivative Runge-Kutta-Nyström method with trigonometric coefficients. Numer. Algorithms 70(1), 1-18 (2015)

14. Kalogiratou, Z: Diagonally implicit trigonometrically fitted symplectic Runge-Kutta methods. Appl. Math. Comput. 219(14), 7406-7412 (2013) 
15. Kalogiratou, Z., Monovasilis, T., Simos, T.E.: A sixth order symmetric and symplectic diagonally implicit Runge-Kutta method. In: International Conference of Computational Metho. vol. 1618, pp. 833-838. Am. Inst. of Phys., New York (2014)

16. Li, J., Deng, S., Wang, X.: Extended explicit pseudo two-step RKN methods for oscillatory systems $y^{\prime \prime}+M y=f(y)$. Numer. Algorithms 78, 673-700 (2018)

17. Li, J., Wang, X., Deng, S., Wang, B.: Trigonometrically-fitted symmetric two-step hybrid methods for oscillatory problems. J. Comput. Appl. Math. 344, 115-131 (2018)

18. Moo, K.W., Senu, N., Ismail, F., Arifin, N.M.: A zero-dissipative phase-fitted fourth order diagonally implicit Runge-Kutta-Nyström method for solving oscillatory problems. Math. Probl. Eng. 2, 1-8 (2014)

19. Paternoster, B.: Runge-Kutta(-Nyström) methods for ODEs with periodic solutions based on trigonometric polynomials. Appl. Numer. Math. 28, 401-412 (1998)

20. Qin, M.Z., Zhu, W.J.: Canonical Runge-Kutta-Nyström methods for second order ordinary differential equations. Comput. Math. Appl. 22(9), 85-95 (1991)

21. Sanz-Serna, J.M.: Symplectic integrators for Hamiltonian problems: an overview. Acta Numer. 1, 243-286 (1992)

22. Sanz-Serna, J.M., Calvo, M.P.: Numerical Hamiltonian Problems. Chapman \& Hall, London (1994)

23. Senu, N., Suleiman, M., Ismail, F., Othman, M.: A new diagonally implicit Runge-Kutta-Nyström method for periodic IVPs. WSEAS Trans. Math. 9(9), 679-688 (2010)

24. Sharp, P.W., Fine, J.M., Burrage, K.: Two stage and three stage diagonally implicit Runge-Nutta-Nyström methods of orders three and four. IMA J. Numer. Anal. 10, 489-504 (1990)

25. Simos, T.E.: An exponentially-fitted Runge-Kutta method for the numerical integration of initial-value problems with periodic or oscillating solutions. Comput. Phys. Commun. 115, 1-8 (1998)

26. Simos, T.E., Vigo-Aguiar, J.: Exponentially fitted symplectic integrator. Phys. Rev. E 67, 1 (2003)

27. Van der Houwen, P.J, Sommeijer, B.P.: Diagonally implicit Runge-Nutta-Nyström methods for oscillating problems. SIAM J. Numer. Anal. 26(2), 414-429 (1989)

28. Vanden Berghe, G., De Meyer, H., Van Daele, M., Van Hecke, T.: Exponentially fitted Runge-Kutta methods. J. Comput. Appl. Math. 125, 107-115 (2000)

29. Vanden Berghe, G., Van Daele, M., Van de Vyver, H.: Exponential fitted Runge-Kutta methods of collocation type: fixed or variable knot points? J. Comput. Appl. Math. 159, 217-239 (2003)

30. Wang, B., Meng, F., Fang, Y.: Efficient implementation of RKN type Fourier collocation methods for second-order differential equations. Appl. Numer. Math. 119, 164-178 (2017)

31. Wang, B., Yang, H., Meng, F.: Sixth order symplectic and symmetric explicit ERKN schemes for solving multi-frequency oscillatory nonlinear Hamiltonian equations. Calcolo 54, 117-140 (2017)

32. You, X., Chen, B.: Symmetric and symplectic exponentially fitted Runge-Kutta(-Nyström) methods for Hamiltonian problems. Math. Comput. Simul. 94, 76-95 (2013)

33. Zhai, W., Chen, B.: A fourth order implicit symmetric and symplectic exponentially fitted Runge-Kutta-Nyström method for solving oscillatory problems. Numer. Algebra Control Optim. 9(1), 71-84 (2019)

\section{Submit your manuscript to a SpringerOpen ${ }^{\circ}$ journal and benefit from:}

- Convenient online submission

- Rigorous peer review

- Open access: articles freely available online

- High visibility within the field

- Retaining the copyright to your article

Submit your next manuscript at $\gg$ springeropen.com 